\title{
Pretreatment Lymphocyte to Monocyte Ratio as a Prognostic Marker for Advanced Pulmonary Squamous Cell Carcinoma Treated With Chemotherapy
}

\author{
Seigo Minamia, b, Shouichi Ihara ${ }^{\mathrm{a}}$, Kiyoshi Komuta ${ }^{\mathrm{a}}$
}

\begin{abstract}
Background: Lower lymphocyte to monocyte ratio (LMR), higher neutrophil to lymphocyte ratio (NLR) and modified Glasgow prognostic score (mGPS) 2 have been demonstrated as independent prognostic markers for poor prognosis of advanced non-small cell lung cancer (NSCLC). However, little is known about these three markers as prognostic markers for a specific histological subset of NSCLC, squamous cell carcinoma (SCC). This study aimed to evaluate the prognostic significance of LMR, NLR and mGPS for advanced SCC.
\end{abstract}

Methods: We retrospectively collected 107 patients who met the following criteria: pathologically confirmed SCC, chemo-naive patients who had initiated first-line cytotoxic chemotherapy between September 2007 and February 2017 at our institution, and c-stage IIIB, IV or recurrence after curative-intent surgery or thoracic radiotherapy. In order to demonstrate these three markers as significant prognostic factors, we compared overall survival (OS) between two groups divided by LMR, NLR and mGPS 0 - 1 versus 2 , and performed univariate and multivariate Cox proportional hazard analyses.

Results: Groups with low LMR $(<2.07)$ and high NLR $(\geq 5.28)$ experienced shorter OS (LMR: 6.5 versus 15.6 months in median, $\mathrm{P}<$ 0.01 ; NLR: 8.2 versus 15.6 months, $\mathrm{P}<0.01)$ than groups with high LMR $(\geq 2.07)$ and low NLR $(<5.28)$. However, no significant difference was detected in OS between mGPS $0-1$ and 2 (13.0 versus 13.7 months, $\mathrm{P}=0.61)$. As significant poor prognostic factors, our multivariate Cox hazard analysis detected ECOG PS 2 - 4 (hazard ration (HR): 3.09, 95\% confidence interval (CI): $1.77-5.40 ; \mathrm{P}<0.01$ ) and LMR $<2.07$ (HR: 0.39, 95\% CI: $0.21-0.79$; $<<0.01$ ). However, NLR was not selected in the multivariate analysis.

Conclusion: LMR is an independent prognostic factor for advanced pulmonary SCC. Neither NLR nor mGPS is useful as prognostic factor for this histology. The optimal prognostic markers may differ from

Manuscript submitted May 10, 2018, accepted May 29, 2018

aDepartment of Respiratory Medicine, Osaka Police Hospital, 10-31 Kitayama-cho, Tennoji-ku, Osaka 543-0035, Japan

${ }^{b}$ Corresponding Author: Seigo Minami, Department of Respiratory Medicine, Osaka Police Hospital, 10-31 Kitayama-cho, Tennoji-ku, Osaka-City, Osaka 543-0035, Japan. Email: seigominami@oph.gr.jp

doi: https://doi.org/10.14740/jocmr3490w each subset of NSCLC.

Keywords: Lymphocyte to monocyte ratio; Neutrophil to lymphocyte ratio; Modified Glasgow prognostic score; Squamous cell carcinoma; Non-small cell lung cancer; First-line chemotherapy; Overall survival; Progression-free survival

\section{Introduction}

Squamous cell carcinoma (SCC) accounted for $30.3 \%$ in males and $13.0 \%$ in females of all lung cancer cases during 2005 2008 in Osaka, Japan [1]. Proportion of this histology in lung cancer was similar in the USA, approximately $30 \%$ in men and $20 \%$ in women in 2010 [2]. Incidence rate of SCC has gradually declined in both Japan and the USA $[1,2]$.

SCC had not been favored by development of breakthrough drugs until immune-checkpoint inhibitors (ICIs) recently became available. In contrast, non-squamous nonsmall cell lung cancer (NSCLC) enjoyed some successful opportunities for various tyrosine kinase inhibitors according to genetic information, pemetrexed maintenance therapy and angiogenesis inhibitors. Nowadays, chemotherapeutic strategies vary depending on histological subtypes. For patients with advanced SCC, conventional cytotoxic chemotherapy still play an important role. For untreated patients with tumor proportion score of $\mathrm{PD}-\mathrm{L} 1$ expression $<50 \%$, platinum-based combination is still recommended as the standard first-line regimen.

Besides pathological factors, host-related factors, particularly the systemic inflammatory response (SIR), contribute to development and progression of tumors [3-5]. Inflammatory cells such as macrophage, neutrophil, monocyte, lymphocyte and other cells play a vital role in the tumor microenvironment. There is now a considerable body of evidence of the prognostic significance of various SIR-based scoring systems. These evidences include SIR-based markers using C-reactive protein (CRP), serum albumin concentration, white blood cell differentiation and count. Both lymphocyte to monocyte ratio (LMR) and neutrophil to lymphocyte ratio (NLR) are well known as circulating blood cell-based markers. Neutrophils and monocytes promote tumor development by means of various mechanisms $[6,7]$, while lymphocytes regress cancer cells. 


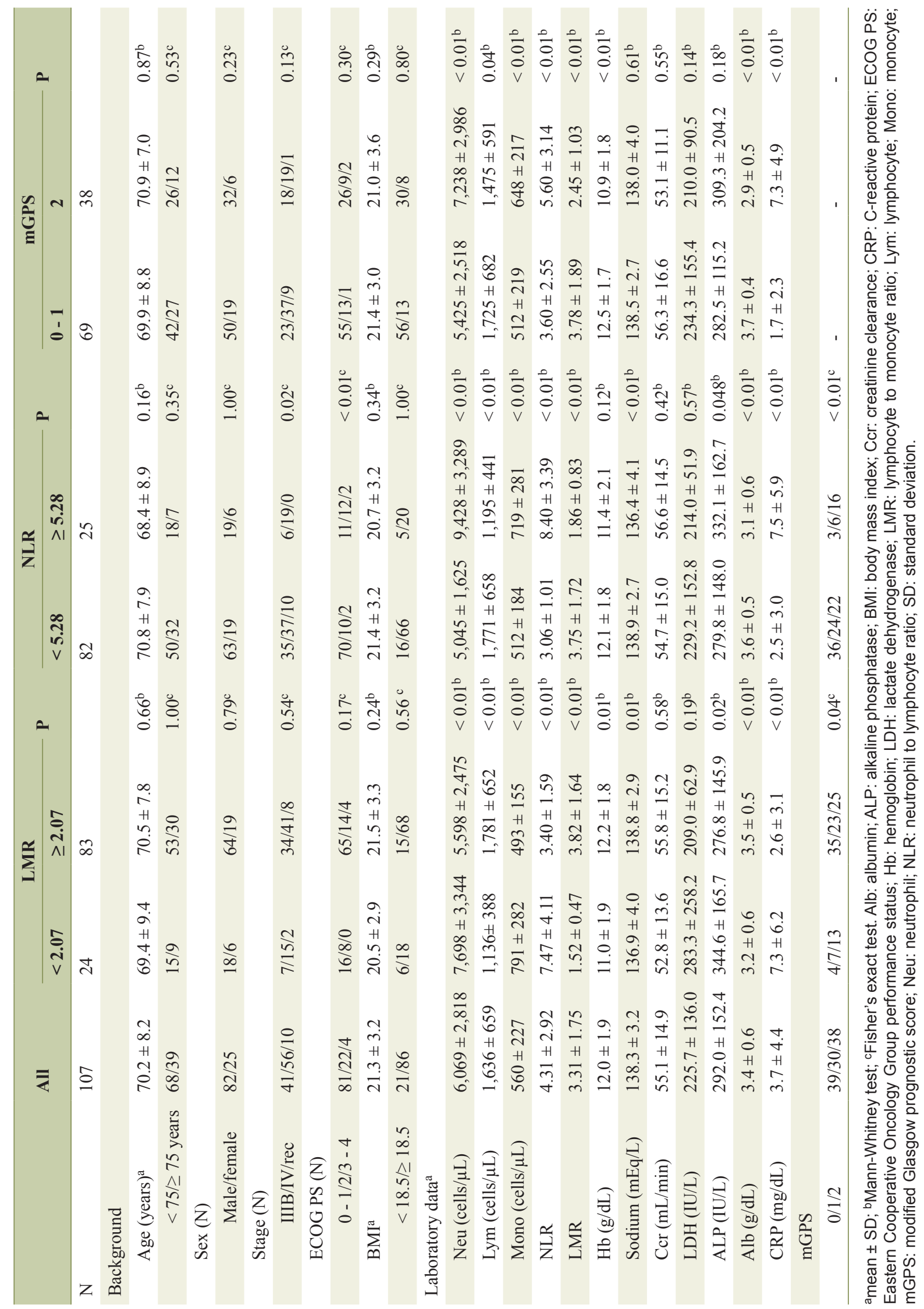


Furthermore, elevated NLR and low LMR reflect the extent of tumor progression. On the other hand, concentration of serum albumin, the main plasma protein, indicates the nutritional status of the patient. Thus, modified Glasgow prognostic score (mGPS) categorizes patients into three classes based on CRP and serum albumin concentration. This score reflects not only host-related SIR status but also nutritional status. In various types of cancers, including advanced NSCLC, higher NLR [8$10]$, lower LMR $[11,12]$ and Glasgow prognostic score (GPS) class $2[13,14]$ are independent prognostic markers for shorter survival. Our previous studies also demonstrated that NLR for NSCLC with active EGFR mutation [15], LMR and mGPS for adenocarcinoma without any driver mutation, and mGPS for small cell lung carcinoma [16] were significant prognostic factors. However, it is not clear that these three prognostic markers are also useful for patients with advanced SCC.

Thus, the aim of this retrospective study was to evaluate the prognostic significance of LMR, NLR and mGPS for advanced SCC.

\section{Patients and Methods}

\section{Patients, study design and data analysis}

This study retrospectively collected 107 patients who met the following inclusion criteria: 1) SCC, confirmed histologically or cytologically; 2) patients who had started the first-line cytotoxic chemotherapy between September 2007 and February 2017; 3) c-stage IIIB-IV based on the seventh TNM classification of lung cancer by the Union for International Cancer Control (UICC) [17], or recurrence after curative thoracic surgery or radiotherapy without adjuvant chemotherapy; 4) laboratory blood data of serum albumin concentration, CRP, absolute counts of neutrophil, lymphocyte and monocyte within 1 week of the first day of the first cycle. Pembrolizumab was approved as the first-line regimen for a specific population of NSCLC by Japanese medical insurance in December 2016, and has been available in our hospital since March 2017. The methods, classifications and definitions of clinical profiles, creatinine clearance (Ccr), NLR, LMR, mGPS, response rate (RR), disease control rate (DCR), progression-free survival (PFS) and overall survival (OS) followed those of our previous studies [15, 16]. The data cutoff was December 31, 2017. The Osaka Police Hospital Ethics Committee approved this study and waiver of the written informed consents because of the anonymous and retrospective data.

\section{Data analysis}

This study adopted the same statistical methods in our previous studies $[15,16]$, as follows: the mean \pm standard deviation (SD) of the continuous data, the frequency of the categorical data, the median ( $95 \%$ confidence intervals (CIs) of the survival data, Fisher's exact test of the relative frequencies, MannWhitney $U$ test of the continuous variables, Spearman's rankorder test of correlation between non-parametric data, Cutoff
Finder (http://molpath.charite.de/cutoff/) [18] to determine the optimal cutoff points of LMR and NLR and stratify our patients into two groups, Kaplan-Meier method and log-rank test for evaluation of PFS and OS, Cox proportional hazard analyses for investigation of independent prognostic factors, EZR (Saitama Medical Center, Jichi Medical University, Saitama, Japan; http://www.jichi.ac.jp/saitama-sct/SaitamaHP.files/statmed.html) [19] used for all statistical analyses. All the factors with $\mathrm{P}$ value $<0.2$ in the preceding univariate analyses were incorporated into the subsequent multivariate analysis. $P$ value $<0.05$ was defined as statistically significant.

\section{Results}

In this study, $107 \mathrm{SCC}$ patients were collected. As of data cutoff (December 31, 2017), 60, 9 and 6 patients were confirmed dead at our hospital, at other medical institutions and at home, respectively. Twenty patients moved to other medical institutions for end-of-life care, but we could not confirm their death. One was missing. We were still following 11 patients. All patients discontinued the first-line chemotherapy, because of documented PD in 35, completion of pre-defined courses in 26 , deteriorated comorbidity or general condition in 22 , adverse effects in 20, patients' refusal in 2 and unknown reason in 2 . Among 64 patients who had proceeded into the secondline chemotherapy, seven patients and one received nivolumab and pembrolizumab, respectively. In this study, 81 and 93 patients had been dead or lost to follow-up until December 2015 and February 2017, when nivolumab and PD-L1 immunohistochemistry assay were approved by Japanese medical insurance, respectively.

Backgrounds, pretreatment laboratory data, treatment details and efficacy of 107 patients are shown in Tables 1 and 2, respectively. LMR and NLR were significantly and inversely correlated $(\mathrm{r}=-0.75, \mathrm{P}<0.01)$. As the optimal cutoff for LMR and NLR, 2.07 and 5.28 were selected by the Cutoff Finder, respectively (Fig. 1). We divided 107 patients into higher and lower groups according to these cutoff points. There were 9 patients in LMR $<2.07+$ NLR $<5.28$ group, 15 in LMR $<$ $2.07+\mathrm{NLR} \geq 5.28,73$ in $\mathrm{LMR} \geq 2.07+\mathrm{NLR}<5.28$ and 10 in $\mathrm{LMR} \geq 2.07+\mathrm{NLR} \geq 5.28$.

High LMR and low NLR groups provided higher RR, longer PFS and OS, and higher treatment rate of second-line chemotherapy than low LMR and high NLR groups. However, there was no significant difference in RR, DCR, PFS and OS between mGPS 0 - 1 and 2. (Table 2 and Fig. 2).

According to our univariate Cox hazard analysis, Eastern Cooperative Oncology Group performance status (ECOG PS) 2 - 4 (HR: 2.92, 95\% CI: 1.76 - 4.86; P < 0.01), higher CRP level (HR: 1.09, 95\% CI: $1.03-1.15 ; \mathrm{P}=0.01$ ), NLR $\geq 5.28$ (HR: $2.80,95 \%$ CI: $1.62-4.83$; P $<0.01$ ), LMR $<2.07$ (HR: 8.00, 95\% CI: $0.18-0.54$; $\mathrm{P}<0.01)$ were significant unfavorable prognostic factors. mGPS was not chosen as a significant prognostic factor (Table 3). In the subsequent multivariate analysis, ECOG PS 2 - 4 (HR: 3.09, 95\% CI: 1.77 - 5.40; P $<0.01$ ) and LMR < 2.07 (HR: 0.39, 95\% CI: $0.21-0.79$; $\mathrm{P}<$ $0.01)$ were significant poor prognostic factors. NLR was not 


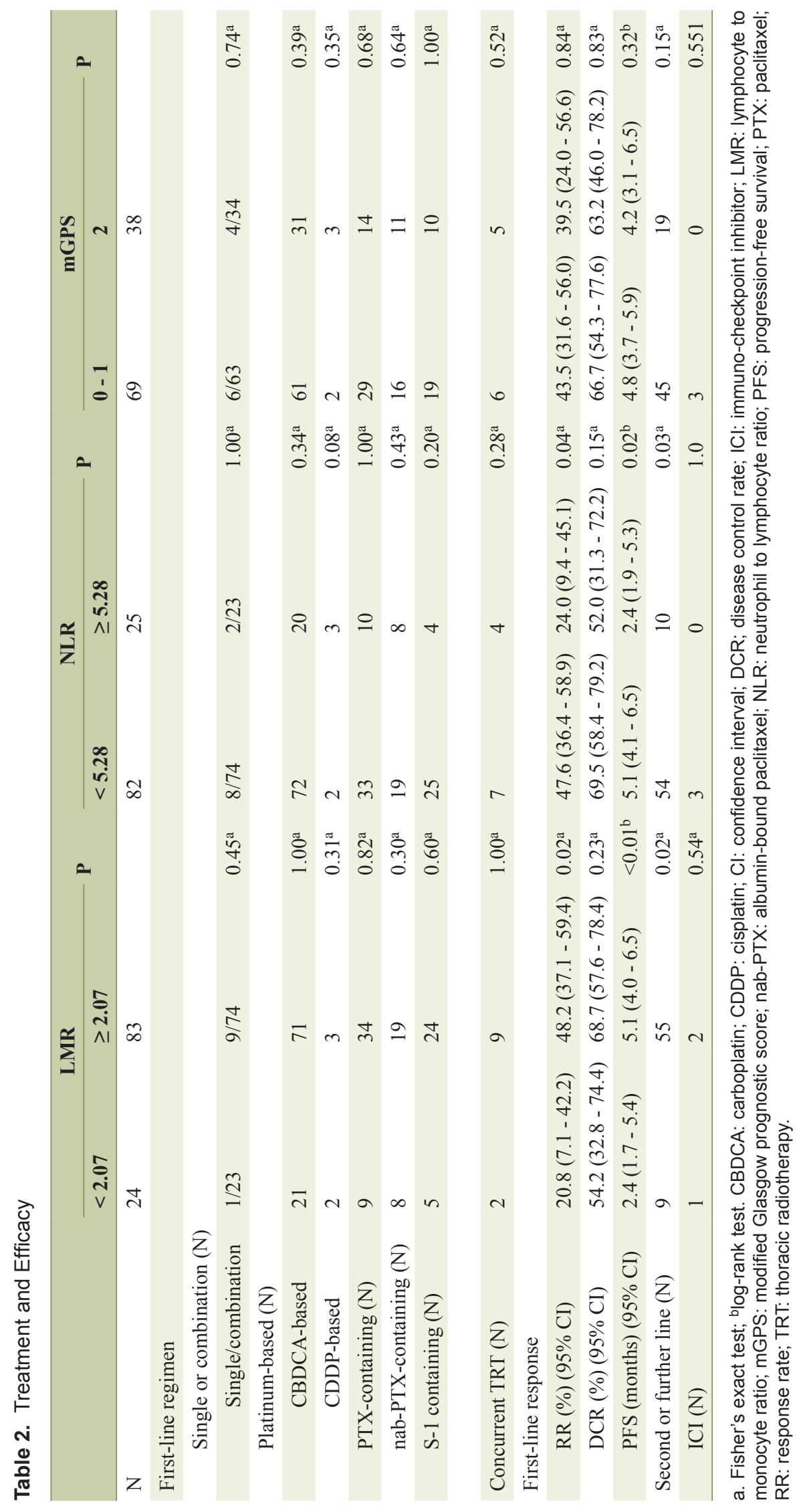


a Significant $(p<0.05)$ tests: 86 out of $87(98.9 \%)$

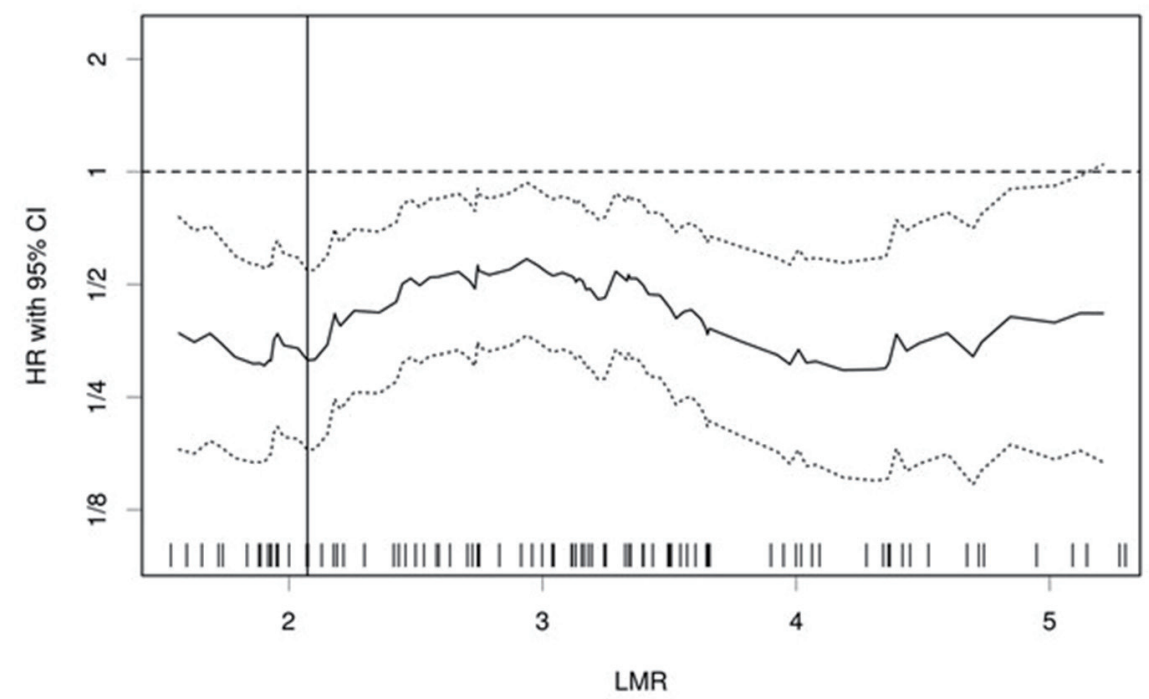

b Significant $(p<0.05)$ tests: 75 out of $87(86.2 \%)$

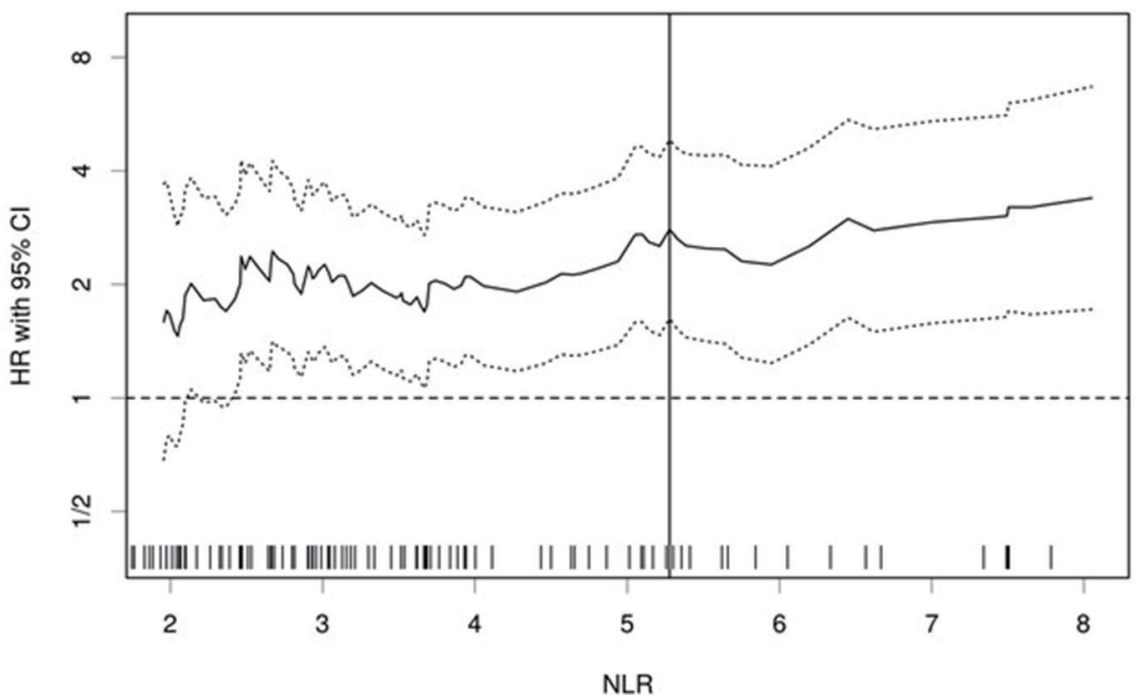

Figure 1. Hazard ratios and cutoff points of LMR and NLR for overall survival of patients with pulmonary squamous cell carcinoma. (a) LMR; (b) NLR. The plots were determined using Cutoff Finder. The vertical lines are the optimal cutoff points providing the most significant split (log-rank test). LMR: lymphocyte to monocyte ratio; NLR: neutrophil to lymphocyte ratio; HR: hazard ratio; OS: overall survival.

selected as a significant prognostic factor (Table 4).

\section{Discussion}

This study was marked by exclusive focus on SCC, a specific histological subset of NSCLC. This was the first study that showed LMR as a significant prognostic marker for SCC.

LMR was an independent prognostic factor for OS of SCC patients, while NLR was not. These results were similar to our previous study, but were different from another our study. According to our previous multivariate analyses, LMR, but not NLR, was an independent prognostic factor for advanced adenocarcinoma with wild-type epidermal growth factor receptor (EGFR). Two previous studies for advanced and unselected NSCLC $[12,20]$ and another study for advanced EGFR-mutated NSCLC treated with EGFR tyrosine kinase inhibitors (TKIs) [21] detected LMR as independent prognostic factors for OS. On the contrary, according to another our study of patients with NSCLC harboring activated EGFR mutation who 

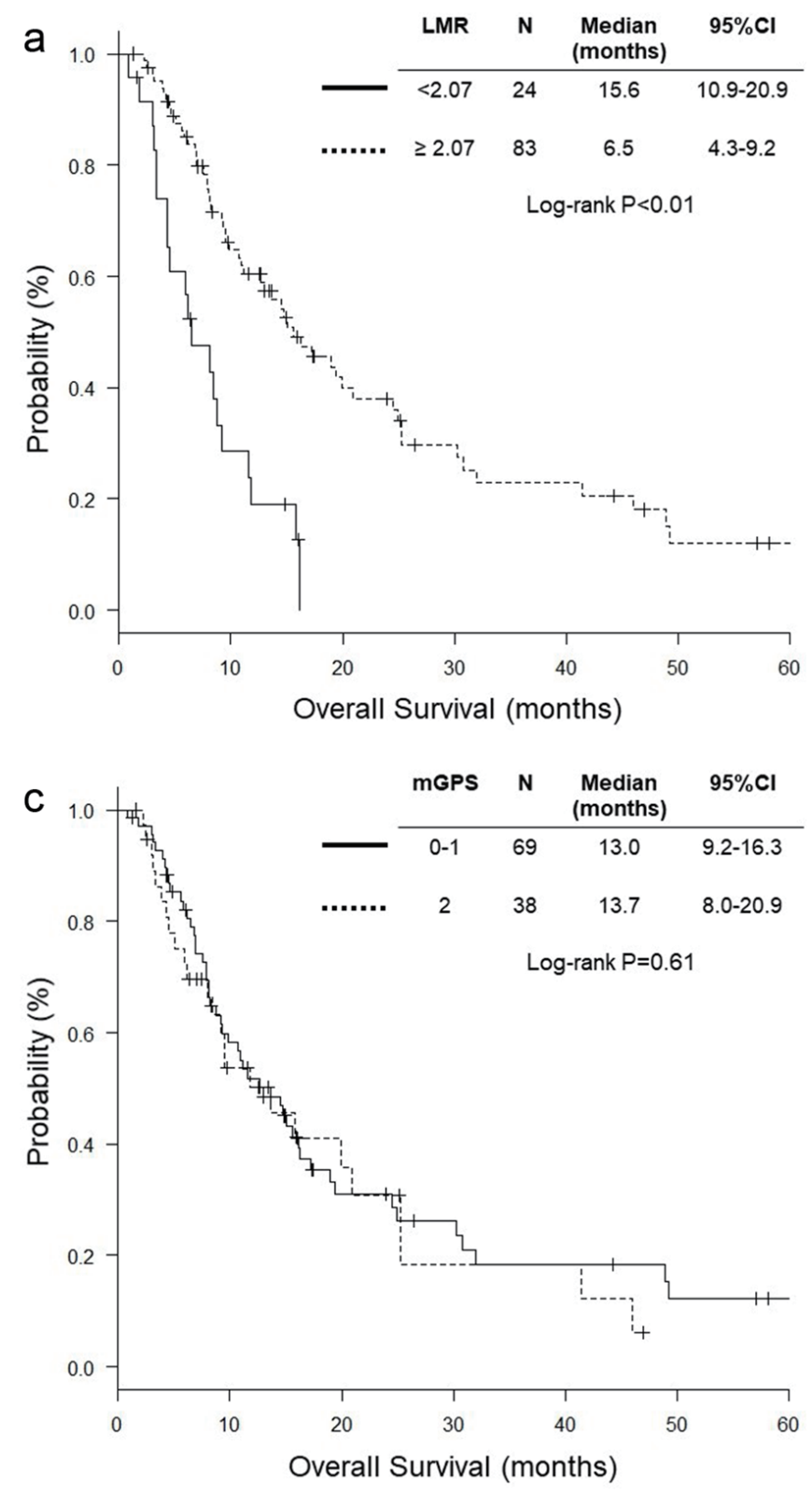

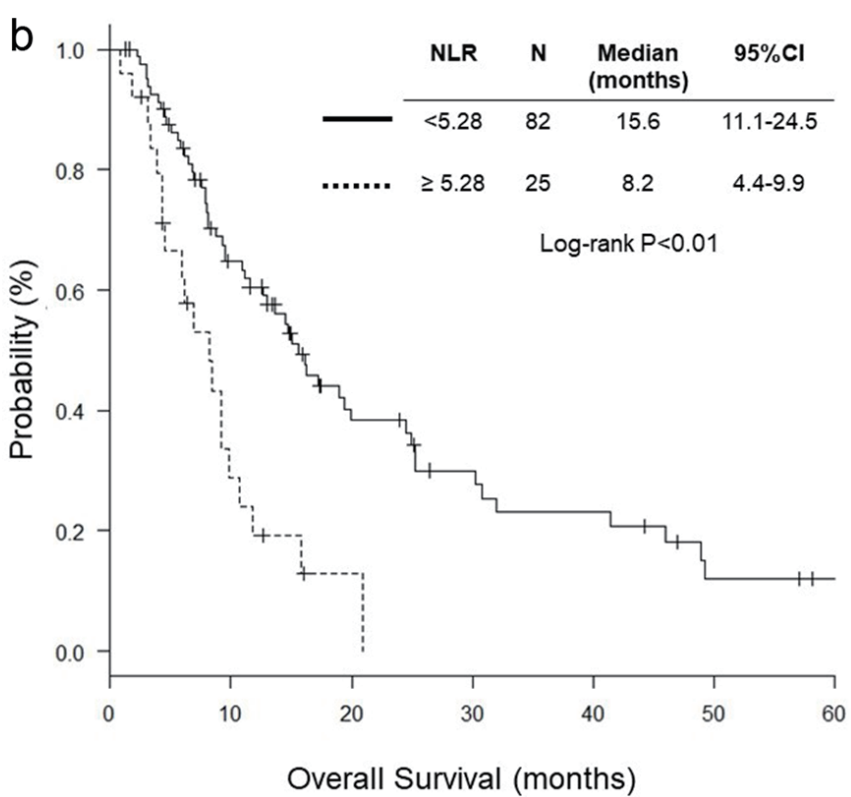

Figure 2. Kaplan-Meier survival curves of overall survival according to LMR, NLR and mGPS. (a) LMR; (b) NLR; (c) mGPS. LMR: lymphocyte to monocyte ratio; mGPS: modified Glasgow prognostic score; NLR: neutrophil to lymphocyte ratio.

had been treated with EGFR-TKIs, NLR, but not LMR, was an independent prognostic factor for this selected population. Compared with LMR, more lines of evidence indicated NLR as an independent prognostic factor for advanced NSCLC. Our series of studies of various subsets of NSCLC suggested that the optimal systemic inflammation marker is different by histological and genetic subsets even in NSCLC.

Interestingly, mGPS was not selected as an independent prognostic factor for SCC patients, based on our univariate Cox hazard analysis and Kaplan-Meier survival curves. In contrast, our previous studies demonstrated mGPS as significant prognostic factors for advanced adenocarcinoma with wild-type EGFR and small cell lung cancer [16]. Several studies also showed clinical utility of mGPS for advanced NSCLC [14, 22-25]. mGPS is an combined index of systemic inflammation and malnutrition, while LMR and NLR are simple indexes of systemic inflammation. Thus, mGPS may play different roles as a prognostic factor according to subsets of NSCLC.

We have to pay attention to some limitations in this study. First, our small sample size might fail to detect some potential biomarkers, especially NLR, as significant prognostic markers. Second, sample bias should be noted because of our singleinstitutional and retrospective study design. Third, our study did not reflect newly developed ICIs. Only a small number of our patients received PD-L1 immunohistochemistry assay and ICIs treatment. NLR has been indicated as a prognostic mark- 
Table 3. Univariate Cox Proportional Hazard Analysis of Factors Associated With Overall Survival

\begin{tabular}{|c|c|c|c|}
\hline & HR & $95 \% \mathrm{CI}$ & $\mathbf{P}$ \\
\hline \multicolumn{4}{|l|}{ Age (years) } \\
\hline$<75$ versus $\geq 75$ & 1.20 & $0.75-1.93$ & 0.45 \\
\hline \multicolumn{4}{|l|}{ Sex } \\
\hline Female versus male & 1.62 & $0.90-2.91$ & 0.11 \\
\hline \multicolumn{4}{|l|}{ Distant metastasis } \\
\hline No versus yes & 1.47 & $0.92-2.37$ & 0.11 \\
\hline \multicolumn{4}{|l|}{ ECOG PS } \\
\hline $0-1$ versus $2-4$ & 2.92 & $1.76-4.86$ & $<0.01$ \\
\hline \multicolumn{4}{|l|}{ BMI } \\
\hline$\geq 18.5$ versus $<18.5$ & 1.42 & $0.80-2.52$ & 0.23 \\
\hline Hemoglobin (g/dL) & 0.96 & $0.85-1.09$ & 0.55 \\
\hline $\operatorname{Ccr}(\mathrm{mL} / \mathrm{min})(/ 10)$ & 1.02 & $0.88-1.18$ & 0.81 \\
\hline \multicolumn{4}{|l|}{ Sodium $(\mathrm{mEq} / \mathrm{L})(/ 10)$} \\
\hline$\geq 135$ versus $<135$ & 0.91 & $0.45-1.82$ & 0.78 \\
\hline LDH (IU/L) (/100) & 1.03 & $0.85-1.23$ & 0.80 \\
\hline $\operatorname{ALP}(\mathrm{IU} / \mathrm{L})(/ 100)$ & 1.05 & $0.90-1.21$ & 0.55 \\
\hline Albumin (g/dL) & 0.72 & $0.49-1.07$ & 0.10 \\
\hline CRP (mg/dL) & 1.09 & $1.03-1.15$ & $<0.01$ \\
\hline \multicolumn{4}{|l|}{ NLR } \\
\hline$<5.28$ versus $\geq 5.28$ & 2.80 & $1.62-4.83$ & $<0.01$ \\
\hline \multicolumn{4}{|l|}{ LMR } \\
\hline$\geq 2.07$ versus $<2.07$ & 8.00 & $0.18-0.54$ & $<0.01$ \\
\hline \multicolumn{4}{|l|}{ mGPS } \\
\hline 0,1 versus 2 & 1.14 & $0.70-1.85$ & 0.61 \\
\hline
\end{tabular}

ALP: alkaline phosphatase; BMI: body mass index; Ccr: creatinine clearance; Cl: confidence interval; CRP: C-reactive protein; ECOG PS: Eastern Cooperative Oncology Group performance status; HR: hazard ratio; LDH: lactate dehydrogenase; LMR: lymphocyte to monocyte ratio; mGPS: modified Glasgow prognostic score; NLR: neutrophil to lymphocyte ratio.

er for patients with lung cancer treated with nivolumab [26], while there is still little evidence of LMR for ICI treatment. Thus, our study could not elucidate the association of LMR with PD-L1 expression and ICI efficacy.

\section{Conclusions}

LMR is an independent prognostic factor for pulmonary SCC. Neither NLR nor mGPS is useful as prognostic factor for this histology. The optimal prognostic markers may differ from each subset of NSCLC.

\section{Acknowledgments}

We are grateful to Sung-Ho Kim, Yoshimi Noda, Yuki Naka-
Table 4. Multivariate Cox Proportional Hazard Analysis of Factors Associated With Overall Survival

\begin{tabular}{|c|c|c|c|}
\hline & HR & $95 \%$ CI & $\mathbf{P}$ \\
\hline \multicolumn{4}{|l|}{ Sex } \\
\hline Female versus male & 1.32 & $0.71-2.43$ & 0.38 \\
\hline \multicolumn{4}{|l|}{ Distant metastasis } \\
\hline No versus yes & 1.54 & $0.90-2.60$ & 0.11 \\
\hline \multicolumn{4}{|l|}{ ECOG PS } \\
\hline $0-1$ versus $2-4$ & 3.09 & $1.77-5.40$ & $<0.01$ \\
\hline Albumin (g/dL) & 1.24 & $0.76-2.04$ & 0.39 \\
\hline CRP (mg/dL) & 1.03 & $0.96-1.10$ & 0.42 \\
\hline \multicolumn{4}{|l|}{ NLR } \\
\hline$<5.28$ versus $\geq 5.28$ & 1.37 & $0.67-2.79$ & 0.39 \\
\hline \multicolumn{4}{|l|}{ LMR } \\
\hline$<2.07$ versus $\geq 2.07$ & 0.41 & $0.21-0.79$ & $<0.01$ \\
\hline
\end{tabular}

Cl: confidence interval; CRP: C-reactive protein; ECOG PS: Eastern Cooperative Oncology Group performance status; HR: hazard ratio; LMR: lymphocyte to monocyte ratio; NLR: neutrophil to lymphocyte ratio.

tani, Kanako Nishimatsu, Shouko Ikuta, Saori Ikebe, Hideyasu Okada and Suguru Yamamoto at the Department of Respiratory Medicine, Osaka Police Hospital for their detailed medical records, diagnosis, treatment and care of their patients.

\section{Conflict of Interest}

The authors declare that they have no conflict of interests.

\section{Grant Support}

None.

\section{References}

1. Kinoshita FL, Ito Y, Nakayama T. Trends in lung cancer incidence rates by histological type in 1975-2008: a population-based study in Osaka, Japan. J Epidemiol. 2016;26(11):579-586.

2. Meza R, Meernik C, Jeon J, Cote ML. Lung cancer incidence trends by gender, race and histology in the United States, 1973-2010. PLoS One. 2015;10(3):e0121323.

3. Coussens LM, Werb Z. Inflammation and cancer. Nature. 2002;420(6917):860-867.

4. Mantovani A, Romero P, Palucka AK, Marincola FM. Tumour immunity: effector response to tumour and role of the microenvironment. Lancet. 2008;371(9614):771783.

5. Proctor MJ, Talwar D, Balmar SM, O'Reilly DS, Foulis AK, Horgan PG, Morrison DS, et al. The relationship between the presence and site of cancer, an inflammation- 
based prognostic score and biochemical parameters. Initial results of the Glasgow Inflammation Outcome Study. Br J Cancer. 2010;103(6):870-876.

6. Augier S, Ciucci T, Luci C, Carle GF, Blin-Wakkach C, Wakkach A. Inflammatory blood monocytes contribute to tumor development and represent a privileged target to improve host immunosurveillance. J Immunol. 2010;185(12):7165-7173.

7. Powell DR, Huttenlocher A. Neutrophils in the tumor microenvironment. Trends Immunol. 2016;37(1):41-52.

8. Gu XB, Tian T, Tian XJ, Zhang XJ. Prognostic significance of neutrophil-to-lymphocyte ratio in non-small cell lung cancer: a meta-analysis. Sci Rep. 2015;5:12493.

9. Peng B, Wang YH, Liu YM, Ma LX. Prognostic significance of the neutrophil to lymphocyte ratio in patients with non-small cell lung cancer: a systemic review and meta-analysis. Int J Clin Exp Med. 2015;8(3):3098-3106.

10. Yin Y, Wang J, Wang X, Gu L, Pei H, Kuai S, Zhang Y, et al. Prognostic value of the neutrophil to lymphocyte ratio in lung cancer: A meta-analysis. Clinics (Sao Paulo). 2015;70(7):524-530.

11. Hu P, Shen H, Wang G, Zhang P, Liu Q, Du J. Prognostic significance of systemic inflammation-based lymphocyte- monocyte ratio in patients with lung cancer: based on a large cohort study. PLoS One. 2014;9(9):e108062.

12. Adam H, Hug S, Bosshard G. Chemotherapy near the end of life: a retrospective single-centre analysis of patients' charts. BMC Palliat Care. 2014;13:26.

13. Jiang AG, Chen HL, Lu HY. The relationship between Glasgow Prognostic Score and serum tumor markers in patients with advanced non-small cell lung cancer. BMC Cancer. 2015;15:386.

14. Leung EY, Scott HR, McMillan DC. Clinical utility of the pretreatment glasgow prognostic score in patients with advanced inoperable non-small cell lung cancer. J Thorac Oncol. 2012;7(4):655-662.

15. Minami S, Ogata Y, Ihara S, Yamamoto S, Komuta K. Neutrophil-to-lymphocyte ratio predicts overall survival of advanced non-small cell lung cancer harboring mutant epidermal growth factor receptor. World J Oncol. 2017;8(6):180-187.

16. Minami S, Ogata Y, Ihara S, Yamamoto S, Komuta K. Pretreatment Glasgow prognostic score and prognostic nutritional index predict overall survival of patients with advanced small cell lung cancer. Lung Cancer (Auckl). 2017;8:249-257.
17. Rami-Porta R, Crowley JJ, Goldstraw P. The revised TNM staging system for lung cancer. Ann Thorac Cardiovasc Surg. 2009;15(1):4-9.

18. Budczies J, Klauschen F, Sinn BV, Gyorffy B, Schmitt WD, Darb-Esfahani S, Denkert C. Cutoff Finder: a comprehensive and straightforward Web application enabling rapid biomarker cutoff optimization. PLoS One. 2012;7(12):e51862.

19. Kanda Y. Investigation of the freely available easy-touse software 'EZR' for medical statistics. Bone Marrow Transplant. 2013;48(3):452-458.

20. Chang YP, Chen YM, Lai CH, Lin CY, Fang WF, Huang $\mathrm{CH}$, Li SH, et al. The impact of de novo liver metastasis on clinical outcome in patients with advanced non-smallcell lung cancer. PLoS One. 2017;12(6):e0178676.

21. Chen YM, Lai CH, Chang HC, Chao TY, Tseng CC, Fang WF, Wang CC, et al. Baseline and trend of lymphocyte-to-monocyte ratio as prognostic factors in epidermal growth factor receptor mutant non-small cell lung cancer patients treated with first-line epidermal growth factor receptor tyrosine kinase inhibitors. PLoS One. 2015;10(8):e0136252.

22. Forrest LM, McMillan DC, McArdle CS, Angerson WJ, Dunlop DJ. Comparison of an inflammation-based prognostic score (GPS) with performance status (ECOG) in patients receiving platinum-based chemotherapy for inoperable non-small-cell lung cancer. Br J Cancer. 2004;90(9):1704-1706.

23. Jiang AG, Chen HL, Lu HY. Comparison of Glasgow prognostic score and prognostic index in patients with advanced non-small cell lung cancer. J Cancer Res Clin Oncol. 2015;141(3):563-568.

24. Jiang AG, Lu HY. The Glasgow prognostic score as a prognostic factor in patients with advanced non-small cell lung cancer treated with cisplatin-based first-line chemotherapy. J Chemother. 2015;27(1):35-39.

25. Umihanic S, Umihanic S, Jamakosmanovic S, Brkic S, Osmic M, Dedic S, Ramic N. Glasgow prognostic score in patients receiving chemotherapy for non-small-cell lung cancer in stages IIIb and IV. Med Arch. 2014;68(2):8385.

26. Bagley SJ, Kothari S, Aggarwal C, Bauml JM, Alley EW, Evans TL, Kosteva JA, et al. Pretreatment neutrophil-tolymphocyte ratio as a marker of outcomes in nivolumabtreated patients with advanced non-small-cell lung cancer. Lung Cancer. 2017;106:1-7. 